Bull. Korean Math. Soc. 47 (2010), No. 1, pp. 167-178

DOI 10.4134/BKMS.2010.47.1.167

\title{
NOTES ON CRITICAL ALMOST HERMITIAN STRUCTURES
}

\author{
Jung Chan Lee, Jeong Hyeong Park, and Kouei Sekigawa
}

ABstract. We discuss the critical points of the functional $\mathcal{F}_{\lambda, \mu}(J, g)=$ $\int_{M}\left(\lambda \tau+\mu \tau^{*}\right) d v_{g}$ on the spaces of all almost Hermitian structures $\mathcal{A H}(M)$ with $(\lambda, \mu) \in \mathbb{R}^{2}-(0,0)$, where $\tau$ and $\tau^{*}$ being the scalar curvature and the $*$-scalar curvature of $(J, g)$, respectively. We shall give several characterizations of Kähler structure for some special classes of almost Hermitian manifolds, in terms of the critical points of the functionals $\mathcal{F}_{\lambda, \mu}(J, g)$ on $\mathcal{A H}(M)$. Further, we provide the almost Hermitian analogy of the Hilbert's result.

\section{Introduction}

Let $M$ be a compact orientable smooth manifold of dimension $m$. We denote by $\mathcal{M}(M)$ the set of all Riemannian metrics on $M$ and $\mathcal{M}_{c}(M)=\{g \in$ $\mathcal{M}(M) \mid \operatorname{Vol}(M, g)=c\}$, where $c$ is a positive constant. It is well-known that a Riemannian metric $g \in \mathcal{M}_{c}(M)$ is a critical point of the (so-called) Einstein-Hilbert functional $\mathcal{F}$ on $\mathcal{M}_{c}(M)$ denoted by

$$
\mathcal{F}(g)=\int_{M} \tau d v_{g}
$$

if and only if $g$ is an Einstein metric, where $\tau$ is the scalar curvature of $g$ and $d v_{g}$ is the volume element of $g$ [5]. Now, let $M$ be a compact smooth manifold of dimension $m=2 n$ admitting an almost complex structure. We denote by $\mathcal{A H}(M)$ the set of all almost Hermitian structures on $M$. It is also known that the space $\mathcal{A H}(M)$ is a contractible Frechet space. Let $\tau$ and $\tau^{*}$ be the scalar curvature and the $*$-scalar curvature of $(J, g)$, respectively. Let $(\lambda, \mu) \in \mathbb{R}^{2}-(0,0)$. In [6], Koda introduced and studied the functional $\mathcal{F}_{\lambda, \mu}$ on $\mathcal{A H}(M)$, which is a generalization of the Einstein-Hilbert functional $\mathcal{F}$ in almost Hermitian case, that is defined by

$$
\mathcal{F}_{\lambda, \mu}(J, g)=\int_{M}\left(\lambda \tau+\mu \tau^{*}\right) d v_{g},
$$

Received December 29, 2008.

2000 Mathematics Subject Classification. 53C15, 53C55.

Key words and phrases. critical almost Hermitian structure, Einstein-Hilbert functional.

This work was supported by the Korea Science and Engineering Foundation(KOSEF) grant funded by the Korea Government(MEST) (R01-2008-000-20370-0). 
and discussed the critical points on $\mathcal{A H}(M)$ and

$$
\mathcal{A H}_{c}(M)=\left\{(J, g) \in \mathcal{A H}(M) \mid g \in \mathcal{M}_{c}(M)\right\} .
$$

We here note that, if $(J, g)$ is a critical point of the functional $\mathcal{F}_{\lambda, \mu}$ on $\mathcal{A H}(M)$, then $(J, g)$ is also a critical point of the functional $\mathcal{F}_{s \lambda, s \mu}$ on $\mathcal{A} \mathcal{H}(M)$ for any non-zero real number $s$, and vice versa. In the present paper, we shall reconsider the critical points of the functional $\mathcal{F}_{\lambda, \mu}$ on $\mathcal{A} \mathcal{H}(M)$ and $\mathcal{A H}_{c}(M)$ by using a slightly different method from the ones used by Koda [6], and give conditions for an almost Hermitian manifold to be a Kähler manifold in terms of critical almost Hermitian structures for certain $(\lambda, \mu)$. In the last section, we shall remark on the critical points of the functional $\mathcal{F}_{\lambda, \mu}$ on $\mathcal{A H}_{c}(M)$.

\section{Preliminaries}

In this section, we prepare some fundamental tools which we need in our arguments. Let $M=(M, J, g)$ be a $2 n$-dimensional almost Hermitian manifold with almost Hermitian structure $(J, g)$ and $\Omega$ be the Kähler form of $M$ defined by $\Omega(X, Y)=g(X, J Y)$ for $X, Y \in \mathfrak{X}(M), \mathfrak{X}(M)$ denoting the Lie algebra of all smooth vector fields $X, Y$ on $M$. We assume that $M$ is oriented by the volume form $d v_{g}=\frac{(-1)^{n}}{n !} \Omega^{n}$. We denote by $\nabla, R, \rho$ and $\tau$ the Riemannian connection, the curvature tensor, the Ricci tensor and scalar curvature of $M$, respectively. The curvature tensor is defined by

$$
R(X, Y) Z=\left[\nabla_{X}, \nabla_{Y}\right] Z-\nabla_{[X, Y]} Z
$$

for $X, Y, Z \in \mathfrak{X}(M)$. A tensor field $\rho^{*}$ on $M$ of type $(0,2)$ defined by

$$
\begin{aligned}
\rho^{*}(X, Y) & =\operatorname{tr}(Z \mapsto R(X, J Z) J Y) \\
& =\frac{1}{2} \operatorname{tr}(Z \mapsto R(X, J Y) J Z)
\end{aligned}
$$

is called a Ricci $*$-tensor, for $X, Y, Z \in \mathfrak{X}(M)$, respectively. We denote by $\tau^{*}$ the $*$-scalar curvature of $M$, which is the trace of the linear endomorphism $Q^{*}$ defined by $g\left(Q^{*} X, Y\right)=\rho^{*}(X, Y)$. We remark that $\rho^{*}$ satisfies

$$
\rho^{*}(X, Y)=\rho^{*}(J Y, J X)
$$

for any $X, Y \in \mathfrak{X}(M)$. Thus $\rho^{*}$ is symmetric if and only if $\rho^{*}$ is J-invariant. In this paper, for any orthonormal basis, (resp. local orthonormal frame field) $\left\{e_{i}\right\}_{i=1, \ldots, 2 n}$ at any point $p \in M$ (resp. on a neighborhood of $p$ ), we shall adopt the following notational conventions:

$$
\begin{aligned}
R_{i j k l}=g\left(R\left(e_{i}, e_{j}\right) e_{k}, e_{l}\right), & R_{\bar{i} \bar{j} \bar{k} \bar{l}} & =g\left(R\left(J e_{i}, J e_{j}\right) J e_{k}, J e_{l}\right), \\
\rho_{i j}=\rho\left(e_{i}, e_{j}\right), & \rho_{\bar{i} \bar{j}} & =\rho\left(J e_{i}, J e_{j}\right), \\
\rho_{i j}^{*}=\rho^{*}\left(e_{i}, e_{j}\right), & \rho_{\bar{i} \bar{j}}^{*} & =\rho^{*}\left(J e_{i}, J e_{j}\right), \\
J_{i j}=g\left(J e_{i}, e_{j}\right), & \nabla_{i} J_{j k} & =g\left(\left(\nabla_{e_{i}} J\right) e_{j}, e_{k}\right),
\end{aligned}
$$

and so on, where the Latin indices run over the range $1,2, \ldots, 2 n$. 
Then we have

$$
J_{i j}=-J_{j i}, \quad \Omega_{i j}=-J_{i j}, \quad \nabla_{i} J_{j k}=-\nabla_{i} J_{k j}, \quad \nabla_{i} J_{\bar{j} \bar{k}}=-\nabla_{i} J_{j k} .
$$

We here recall some special classes of almost Hermitian manifolds [4]. We denote by $\mathcal{K}, \mathcal{A K}, \mathcal{N} \mathcal{K}, \mathcal{Q K}, \mathcal{S K}$ and $\mathcal{H}$ the sets of all Kähler manifolds, almost Kähler manifolds, nearly Kähler manifolds, quasi Kähler manifolds, semi Kähler manifolds and Hermitian manifolds, respectively.

(I) $\mathcal{K}$ (Class of Kähler manifolds): An almost Hermitian manifold $M=$ $(M, J, g)$ is called a Kähler manifold if $\nabla J=0$ on $M$.

(II) $\mathcal{A K}$ (Class of almost Kähler manifolds) : An almost Hermitian manifold is called an almost Kähler manifold if $d \Omega=0$ on $M$. The condition $d \Omega=0$ is equivalent to the condition $\nabla_{i} J_{j k}+\nabla_{j} J_{k i}+\nabla_{k} J_{i j}=0$.

(III) $\mathcal{N K}$ (Class of nearly Kähler manifolds): An almost Hermitian manifold is called a nearly Kähler manifold if $\left(\nabla_{X} J\right) Y+\left(\nabla_{Y} J\right) X=0\left(\nabla_{i} J_{j k}+\right.$ $\left.\nabla_{j} J_{i k}=0\right)$ on $M$.

(IV) $\mathcal{Q K}$ (Class of quasi Kähler manifolds): An almost Hermitian manifold is called a quasi Kähler manifold if $\left(\nabla_{X} J\right) Y+\left(\nabla_{J X} J\right) J Y=0\left(\nabla_{i} J_{j k}+\right.$ $\left.\nabla_{\bar{i}} J_{\bar{j} k}=0\right)$ on $M$.

(V) $\mathcal{S K}$ (Class of semi Kähler manifolds): An almost Hermitian manifold is called a semi Kähler manifold if $\delta \Omega=0$ on $M$. The condition $\delta \Omega=0$ is equivalent to the condition $\sum_{a} \nabla_{a} J_{a j}=0$.

(VI) $\mathcal{H}$ (Class of Hermitian manifolds): An almost Hermitian manifold with the integrable almost complex structure $J$ is called a Hermitian manifold. It is well-known that $M$ is a Hermitian manifold if and only if $\left(\nabla_{X} J\right) Y-\left(\nabla_{J X} J\right)(J Y)=0\left(\nabla_{i} J_{j k}-\nabla_{\bar{i}} J_{\bar{j} k}=0\right)$ on $M$.

Now we shall recall some fundamental identities on the above classes (II) (VI).

(II) Let $M=(M, J, g)$ be an almost Kähler manifold. Then, addition to (2.4), we have [3]

$$
\begin{gathered}
\nabla_{\bar{i}} J_{\bar{j} k}+\nabla_{i} J_{j k}=0, \\
2 \sum_{a}\left(\nabla_{a} J_{i j}\right) \nabla_{a} J_{k l} \\
=R_{i j k l}-R_{i j \bar{k} \bar{l}}-R_{\bar{i} \bar{j} k l}+R_{\bar{i} \bar{j} \bar{k} \bar{l}}+R_{\bar{i} \bar{j} \bar{k} l}+R_{\bar{i} \bar{j} k \bar{l}}+R_{i \bar{j} \bar{k} l}+R_{i \bar{j} k \bar{l}} .
\end{gathered}
$$

From (2.6), we have

$$
\rho_{i j}^{*}+\rho_{j i}^{*}-\rho_{i j}-\rho_{\bar{i} \bar{j}}=\sum_{a, b}\left(\nabla_{a} J_{i b}\right) \nabla_{a} J_{j b},
$$

and further

$$
|\nabla J|^{2}=2\left(\tau^{*}-\tau\right) .
$$


(III) Let $M=(M, J, g)$ be a nearly Kähler manifold. Then, it is well-known that the following identities exist on $M[2]$, we have

$$
\begin{gathered}
\nabla_{i} J_{j k}+\nabla_{\bar{i}} J_{\bar{j} k}=0, \\
\rho_{\bar{i} \bar{j}}=\rho_{i j}, \quad \rho_{\overline{i j}}^{*}=\rho_{i j}^{*}, \\
\rho_{i j}-\rho_{i j}^{*}=\sum_{a, b}\left(\nabla_{i} J_{a b}\right) \nabla_{j} J_{a b} .
\end{gathered}
$$

From (2.11), we have

$$
|\nabla J|^{2}=\tau-\tau^{*}(=\text { constant }) .
$$

(V) Let $M=(M, J, g)$ be a semi Kähler manifold. Then, it is well-known that the following identities on $M[2]$ :

$$
\tau-\tau^{*}=\sum\left(\nabla_{k} J_{j i}\right) \nabla_{j} J_{i k} .
$$

Now, let $M=(M, J, g)$ be a 4-dimensional almost Hermitian manifold. Then we have

$$
\tau-\tau^{*}=2 \delta \omega+|\omega|^{2}-\frac{1}{8}|N|^{2},
$$

where $\omega$ is the Lee form of $M$ and $N$ is the Nijenhuis tensor of $M$ [10].

(VI) Let $M=(M, J, g)$ be a 4-dimensional Hermitian manifold. Then from (2.14), in particular, we have

$$
\tau-\tau^{*}=2 \delta \omega+|\omega|^{2} .
$$

Here we note the inclusion relations between the classes (I) (VI).

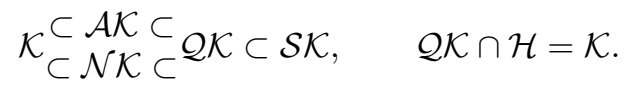

We denote by $\mathcal{K}(M), \mathcal{A K}(M), \mathcal{N} \mathcal{K}(M), \mathcal{Q K}(M), \mathcal{S} \mathcal{K}(M)$ and $\mathcal{H}(M)$, the subsets of $\mathcal{A H}(M)$ of all Kähler structures, almost Kähler structures, nearly Kähler structures, quasi Kähler structures, semi Kähler structures and Hermitian structures on $M$. Then, we may obtain the inclusion relations between these sets corresponding to the above inclusion ones between the classes (I) (IV). In the sequel, we assume always that $M$ is a $2 n(n \geqq 2)$-dimensional compact orientable smooth manifold and $\mathcal{A H}(M) \neq \emptyset$ unless otherwise specified.

\section{Critical points of the functional $\mathcal{F}_{\lambda, \mu}$ on $\mathcal{A H}(M)$}

Let $M$ be a $2 n$-dimensional compact orientable smooth manifold admitting almost complex structure. Let $(J, g) \in \mathcal{A H}(M)$ and consider a smooth curve $(J(t), g(t)) \in \mathcal{A H}(M)$ through $(J, g)$. We shall also call it a (1-parameter) deformation of $(J, g)$. We denote by $\Omega(t)$ the Kähler form of $(J(t), g(t))$ and set $\alpha(t)=\Omega(t)-\Omega$. We denote further by $\nabla^{(t)}, R(t), \rho(t), \rho^{*}(t), \tau(t)$ and $\tau^{*}(t)$ the Riemannian connection, the curvature tensor, the Ricci tensor, the Ricci $*$-tensor, the scalar curvature and the $*$-scalar curvature of 
$(J(t), g(t))$, respectively. Let $\left(U ; x_{1}, \ldots, x_{2 n}\right)$ be a local coordinate system on coordinate neighborhood $U$ of $M$. With respect to the natural frame $\left\{\partial_{i}=\right.$ $\left.\frac{\partial}{\partial x_{i}}\right\}_{i=1, \ldots, 2 n}$, we set $g(t)\left(\partial_{i}, \partial_{j}\right)=g(t)_{i j}, J(t) \partial_{i}=J(t)_{i}{ }^{j} \partial_{j},\left(\nabla_{\partial_{i}}^{(t)} J(t)\right) \partial_{j}=$ $\left(\nabla_{i}^{(t)} J(t)_{j}{ }^{k}\right) \partial_{k}, R(t)\left(\partial_{i}, \partial_{j}\right) \partial_{k}=R(t)_{i j k}{ }^{l} \partial_{l}, \rho(t)\left(\partial_{i}, \partial_{j}\right)=\rho(t)_{i j}, \rho^{*}(t)\left(\partial_{i}, \partial_{j}\right)=$ $\rho^{*}(t)_{i j}, \alpha(t)\left(\partial_{i}, \partial_{j}\right)=\alpha(t)_{i j}$ and $g(t)^{i j}=\left(g(t)_{i j}\right)^{-1}$. In particular, we have $g(0)_{i j}=g_{i j}, J(0)_{i}{ }^{j}=J_{i}{ }^{j}, \nabla_{i}^{(0)} J(0)_{j}{ }^{k}=\nabla_{i} J_{j}{ }^{k}, R(0)_{i j k}{ }^{l}=R_{i j k}{ }^{l}, \rho(0)_{i j}=\rho_{i j}$, $\rho^{*}(0)_{i j}=\rho_{i j}^{*}, \tau(0)=\tau, \tau^{*}(0)=\tau^{*}$ and $\alpha(0)_{i j}=0$.

Further, we set

$$
\left.\frac{d}{d t}\right|_{t=0} g(t)_{i j}=h_{i j},\left.\frac{d}{d t}\right|_{t=0} J(t)_{j}{ }^{i}=K_{j}{ }^{i},\left.\frac{d}{d t}\right|_{t=0} \alpha(t)_{i j}=A_{i j} .
$$

Then we see that $A=\left(A_{i j}\right)$ is a 2 -form, $h=\left(h_{i j}\right)$ is a symmetric $(0,2)$-tensor field on $M$ and we also have

$$
\left.\frac{d}{d t}\right|_{t=0} g(t)^{i j}=-h^{i j}
$$

where we adopt the standard notational convention of tensor analysis: for example $h^{i j}$ means $h^{i j}=g^{i a} g^{j b} h_{a b}$. We denote by $d v_{g(t)}$ the volume of $(M, g(t))$. Then, we have

$$
\left.\frac{d}{d t}\right|_{t=0} d v_{g(t)}=\frac{1}{2}\left(g^{i j} h_{i j}\right) d v_{g} .
$$

From (3.2), we see that the coefficients $\Gamma(t)_{i j}{ }^{k}$ of $\nabla^{(t)}$ satisfy

$$
\left.\frac{d}{d t}\right|_{t=0} \Gamma(t)_{i j}{ }^{k}=\frac{1}{2} g^{k a}\left(\nabla_{i} h_{a j}+\nabla_{j} h_{i a}-\nabla_{a} h_{i j}\right) .
$$

Thus, from (3.4), the derivations of $R(t)_{i j k}{ }^{l}, \rho(t)_{i j}$ and $\tau(t)$ at $t=0$ are given respectively by

$\left.\frac{d}{d t}\right|_{t=0} R(t)_{i j k}{ }^{l}=\frac{1}{2}\left(-R_{i j k}{ }^{a} h_{a}{ }^{l}+R_{i j a}{ }^{l} h_{k}{ }^{a}+\nabla_{i} \nabla_{k} h_{j}{ }^{l}-\nabla_{j} \nabla_{k} h_{i}{ }^{l}-\nabla_{i} \nabla^{l} h_{j k}+\nabla_{j} \nabla^{l} h_{i k}\right)$,

$$
\left.\frac{d}{d t}\right|_{t=0} \rho(t)_{i j}=\frac{1}{2}\left(-R_{a i j}{ }^{b} h_{b}{ }^{a}+\rho_{i a} h_{j}{ }^{a}+\nabla_{a} \nabla_{j} h_{i}{ }^{a}-\nabla_{i} \nabla_{j} h_{a}{ }^{a}-\nabla^{a} \nabla_{a} h_{i j}+\nabla_{i} \nabla_{a} h_{j}{ }^{a}\right),
$$

$$
\left.\frac{d}{d t}\right|_{t=0} \tau(t)=-\rho_{i j} h^{i j}+\nabla^{i} \nabla^{j} h_{i j}-\nabla^{i} \nabla_{i} h_{a}{ }^{a} .
$$

Further, since $(J(t), g(t)) \in \mathcal{A H}(M)$, we have

$$
\begin{gathered}
K_{a}{ }^{i} J_{j}{ }^{a}+J_{a}{ }^{i} K_{j}{ }^{a}=0, \\
h_{i j}=h_{a b} J_{i}{ }^{a} J_{j}{ }^{b}+K_{i a} J_{j}{ }^{a}+J_{i a} K_{j}{ }^{a}, \\
K_{j}{ }^{i}=-h_{a}{ }^{i} J_{j}{ }^{a}-A_{j}{ }^{i},
\end{gathered}
$$




$$
h_{i j}=-h_{a b} J_{i}^{a} J_{j}^{b}+J_{i}^{a} A_{a j}+{J_{j}}^{a} A_{a i} .
$$

From (3.10) and (3.11), we have also

$$
K_{j}{ }^{i}=h_{j}{ }^{a} J_{a}{ }^{i}-A_{b}{ }^{a} J_{a}{ }^{i} J_{j}{ }^{b} .
$$

Conversely, let $(h, A)$ be a pair of a symmetric $(0,2)$-tensor $h=\left(h_{i j}\right)$ and a 2 -form $A=\left(A_{i j}\right)$ satisfying $(3.11)$ and define a $(1,1)$-tensor $K$ by $(3.12)$. Then, we may easily check that the equalities (3.8) and (3.9) hold. This means that for a given $(J, g) \in \mathcal{A H}(M)$ and a pair $(h, A)$ satisfying (3.11), there exists a curve $(J(t), g(t)) \in \mathcal{A H}(M)$ through $(J, g)$ for sufficiently small $t$, where tangent vector at $t=0$ is $(K, h)$. We here introduce several explicit examples of such curves.

(i) Blair-Ianus deformations [1]: The curve $(J(t), g(t))$ through $(J, g)$ which corresponds to $\Omega(t)=\Omega$, where $\Omega$ is the Kähler form of $(J, g)$. The curve $(J(t), g(t))$ can be regarded as a curve in $\mathcal{A H}(M)$ through $(J, g)$ with an initial condition $(h, A)$ such that $A=0$ and $J$-skew invariant $h$.

(ii) The curve $(J(t), g(t))$ through $(J, g)$ with initial condition $(h, A)$ given by $h_{i j}=\frac{1}{2}\left(J_{i}{ }^{a} A_{a j}+J_{j}{ }^{a} A_{a i}\right)$ for any 2 -form $A=\left(A_{i j}\right)$ on $M$.

From (3.3) and (3.4), taking account of (3.10), (3.11), (3.12), we have further

$$
\left.\frac{d}{d t}\right|_{t=0} J(t)^{i j}=-h^{i a} J_{a}^{j}+g^{i a} K_{a}{ }^{j}=A_{a b} J^{i a} J^{j b},
$$

$$
\begin{aligned}
&\left.\frac{d}{d t}\right|_{t=0} \rho^{*}(t)_{i j}= \rho_{i a}^{*} h_{j}{ }^{a}-\frac{1}{2} R_{i u a}{ }^{b} J_{j}{ }^{u} J^{a c} h_{b c}-\frac{1}{2} J^{a b} J_{j}{ }^{c} \nabla_{i} \nabla_{a} h_{b c} \\
&+\frac{1}{2} J^{a b} J_{j}{ }^{c} \nabla_{c} \nabla_{a} h_{b i}+\frac{1}{2}\left(2 J_{j}{ }^{q} \rho^{*}{ }_{i}^{p}-J_{j}{ }^{u} J^{p a} J^{q b} R_{i u a b}\right) A_{p q}, \\
&\left.\frac{d}{d t}\right|_{t=0} \tau^{*}(t)=\rho_{a b}^{*} h^{a b}-J^{i a} J^{j b} \nabla_{a} \nabla_{b} h_{i j}-2 J^{i p} \rho_{i q}^{*} A_{p}{ }^{q} .
\end{aligned}
$$

Now, we are ready to compute the first variation of the functional $\mathcal{F}_{\lambda, \mu}$ on $\mathcal{A H}(M)$. We shall adapt the notational convention (2.3) with respect to a local orthonormal frame field $\left\{e_{i}\right\}_{i=1, \ldots, 2 n}$. By (3.4), (3.7) and (3.15), we have

$$
\begin{aligned}
\left.\frac{d}{d t}\right|_{t=0} \mathcal{F}_{\lambda, \mu}(J(t), g(t))= & \left.\frac{d}{d t}\right|_{t=0} \int_{M}\left(\lambda \tau(t)+\mu \tau^{*}(t)\right) d v_{g(t)} \\
= & \int_{M} \sum\left(-\lambda \rho_{i j}+\mu \rho_{i j}^{*}+\frac{1}{2}\left(\lambda \tau+\mu \tau^{*}\right) g_{i j}\right) h_{i j} d v_{g} \\
& -\mu \int_{M} \sum J_{i a} J_{j b} \nabla_{a} \nabla_{b} h_{i j} d v_{g}+2 \mu \int_{M} \sum \rho_{i j}^{*} A_{i j} d v_{g} \\
= & \int_{M} \sum_{i, j}\left\{\left(-\lambda \rho_{i j}+\mu \rho_{i j}^{*}+\frac{1}{2}\left(\lambda \tau+\mu \tau^{*}\right) g_{i j}\right.\right.
\end{aligned}
$$




$$
\left.\left.-\mu \sum_{a, b} \nabla_{b} \nabla_{a}\left(J_{i a} J_{j b}\right)\right) h_{i j}+2 \mu \rho_{i j}^{*} A_{i j}\right\} d v_{g} .
$$

Here, we get

$$
\begin{aligned}
& \sum_{a, b} \nabla_{b} \nabla_{a}\left(J_{i a} J_{j b}\right)=\sum_{a, b} \nabla_{b}\left(\left(\nabla_{a} J_{i a}\right) J_{j b}+J_{i a} \nabla_{a} J_{j b}\right) \\
= & \sum_{a, b}\left(\nabla_{b} \nabla_{a} J_{i a}\right) J_{j b}+\sum_{a, b}\left(\nabla_{a} J_{i a}\right) \nabla_{b} J_{j b}+\sum_{a, b}\left(\nabla_{b} J_{i a}\right) \nabla_{a} J_{j b}+\sum_{a, b} J_{i a} \nabla_{b} \nabla_{a} J_{j b} \\
= & \sum_{a, b}\left(\nabla_{b} \nabla_{a} J_{i a}\right) J_{j b}+\sum_{a, b}\left(\nabla_{a} J_{i a}\right) \nabla_{b} J_{j b}+\sum_{a, b}\left(\nabla_{b} J_{i a}\right) \nabla_{a} J_{j b} \\
& +\sum_{a, b} J_{i a} \nabla_{a} \nabla_{b} J_{j b}-\sum_{a, b, c} J_{i a} R_{b a j c} J_{c b}-\sum_{a, b, c} J_{i a} R_{b a b c} J_{j c} \\
= & \sum_{a, b}\left(\nabla_{b} \nabla_{a} J_{i a}\right) J_{j b}+\sum_{a, b}\left(\nabla_{a} J_{i a}\right) \nabla_{b} J_{j b}+\sum_{a, b}\left(\nabla_{b} J_{i a}\right) \nabla_{a} J_{j b} \\
& +\sum_{a, b}\left(\nabla_{a} \nabla_{b} J_{j b}\right) J_{i a}-\frac{1}{2} \sum_{a, b, c} J_{i a}\left(R_{a b c j}-R_{a c b j}\right) J_{c b}+\sum_{i a} J_{j c} \rho_{a c} \\
= & \sum_{a, b} J_{j b} \nabla_{b} \nabla_{a} J_{i a}+\sum_{a, b} J_{i a} \nabla_{a} \nabla_{b} J_{j b}+\sum_{a, b}\left(\nabla_{b} J_{i a}\right) \nabla_{a} J_{j b} \\
& +\sum_{a, b}\left(\nabla_{a} J_{i a}\right) \nabla_{b} J_{j b}-\rho_{j i}^{*}+\rho_{i \bar{j}} .
\end{aligned}
$$

We denote by $T=\left(T_{i j}\right)$ the symmetric $(0,2)$-tensor defined by (3.18)

$$
\begin{aligned}
T_{i j}= & -\lambda \rho_{i j}-\mu \rho_{\bar{i} \bar{j}}+\mu\left(\rho_{i j}^{*}+\rho_{j i}^{*}\right)+\frac{1}{2}\left(\lambda \tau+\mu \tau^{*}\right) g_{i j} \\
& -\mu \sum_{a, b}\left(J_{i a} \nabla_{a} \nabla_{b} J_{j b}+J_{j a} \nabla_{a} \nabla_{b} J_{i b}+\left(\nabla_{a} J_{i a}\right) \nabla_{b} J_{j b}+\left(\nabla_{a} J_{j b}\right) \nabla_{b} J_{i a}\right) .
\end{aligned}
$$

Thus, from (3.16), (3.17), (3.18), we have the following.

Lemma 1. $(J, g)$ is a critical point of the functional $\mathcal{F}_{\lambda, \mu}$ on $\mathcal{A H}(M)$ if and only if $(J, g)$ satisfies

$$
\int_{M} \sum_{i, j}\left(T_{i j} h_{i j}+2 \mu \rho_{i j}^{*} A_{i j}\right) d v_{g}=0
$$

for any pair $(h, A)$ of a symmetric $(0,2)$-tensor $h=\left(h_{i j}\right)$ and a 2-form $A=$ $\left(A_{i j}\right)$ satisfying $(3.11)$, where $T=\left(T_{i j}\right)$ is the symmetric $(0,2)$-tensor defined by (3.18).

Now, we recall the following fact due to Blair-Ianus [1]: 
Lemma 2. Let $B=\left(B_{i j}\right)$ be a symmetric $(0,2)$-tensor on $M$. Then

$$
\int_{M} \sum_{i, j} B_{i j} D_{i j} d v_{g}=0
$$

for all symmetric $(0,2)$-tensor $D$ satisfying $D_{i j}+D_{\bar{i} \bar{j}}=0$ if and only if $B$ is $J$-invariant.

Now, let $(J, g)$ be a critical point of $\mathcal{F}_{\lambda, \mu}$ and consider the Blair-Ianus deformation $(J(t), g(t))$ of $(J, g)$. Then, by Lemma 2, we see that the tensor $T$ is $J$-invariant. Next, we consider the deformation $(J(t), g(t))$ of $(J, g)$ of type (ii). Then, we have

$$
\begin{aligned}
& \int_{M} \sum_{i, j}\left(\frac{1}{2} T_{i j} A_{\bar{i} j}+\frac{1}{2} T_{i j} A_{\overline{j i}}+2 \mu \rho_{\overline{i j}}^{*} A_{i j}\right) d v_{g} \\
= & \int_{M} \sum_{i, j}\left(-\frac{1}{2} T_{\bar{i} j}+\frac{1}{2} T_{i \bar{j}}+2 \mu \rho_{\bar{i} j}^{*}\right) A_{i j} d v_{g} \\
= & \int_{M} \sum_{i, j}\left(T_{i \bar{j}}+2 \mu \rho_{\bar{i} j}^{*}\right) A_{i j} d v_{g}=0
\end{aligned}
$$

for all 2-forms $A=\left(A_{i j}\right)$ on $M$. Thus, from (3.20), we have

$$
T_{i \bar{j}}+2 \mu \rho_{i j}^{*}=0 \text {. }
$$

Thus, from (3.21), we see in particular that $\rho^{*}$ is symmetric if $\mu \neq 0$.

Conversely, we assume that $T_{i j}-2 \mu \rho_{\bar{i}}^{*}=0$ and $T=\left(T_{i j}\right)$ is $J$-invariant. Then, for any $(h, A)$ satisfying $(3.11)$, we have

$$
\begin{aligned}
& \int_{M} \sum_{i, j}\left(T_{i j} h_{i j}+2 \mu \rho_{\overline{i j}}^{*} A_{i j}\right) d v_{g} \\
= & 2 \mu \int_{M} \sum_{i, j} \rho_{i j}^{*}\left(h_{i j}-A_{\bar{i} j}\right) d v_{g} \\
= & \mu \int_{M} \sum_{i, j} \rho_{i j}^{*}\left(h_{i j}+h_{\bar{i} \bar{j}}-A_{\bar{i} j}-A_{\bar{j} i}\right) d v_{g} \\
= & 0 .
\end{aligned}
$$

By virtue of (3.11), and hence, from Lemma 1 , we see that $(J, g)$ is a critical point of $\mathcal{F}_{\lambda, \mu}$. Thus, summing up the above arguments, we have finally the following theorem.

Theorem 3. $(J, g)$ is a critical point of the functional $\mathcal{F}_{\lambda, \mu}$ on $\mathcal{A H}(M)$ if and only if $(J, g)$ satisfies $T_{i j}=T_{\bar{i} \bar{j}}$ and $T_{i \bar{j}}+2 \mu \rho_{\bar{i} j}^{*}=0$ (and hence, in particular $\rho^{*}$ is symmetric for a critical point $(J, g)$ of $\left.\mathcal{F}_{\lambda, \mu}(\mu \neq 0)\right)$. 
Remark 1. From (3.18), taking account of the equality $T_{i j}=T_{\bar{i} \bar{j}}$, we may easily check that the equality $T_{i \bar{j}}+2 \mu \rho_{\bar{i} j}^{*}=0$ in Theorem 3 can be rewritten as

$$
\begin{aligned}
& \lambda \rho_{i j}+\mu \rho_{\bar{i} \bar{j}}-\frac{1}{2}\left(\lambda \tau+\mu \tau^{*}\right) g_{i j} \\
& +\mu \sum_{a, b}\left(J_{i a} \nabla_{a} \nabla_{b} J_{j b}+J_{j a} \nabla_{a} \nabla_{b} J_{i b}+\left(\nabla_{a} J_{i a}\right) \nabla_{b} J_{j b}+\left(\nabla_{a} J_{j b}\right) \nabla_{b} J_{i a}\right)=0 .
\end{aligned}
$$

Further, by (3.18), we see that the equality $T_{i j}=T_{\bar{i} \bar{j}}$ is equivalent to the following equality.

$$
\begin{aligned}
& (\lambda-\mu)\left(\rho_{i j}-\rho_{\bar{i} \bar{j}}\right)+\mu\left(\sum_{b} \nabla_{i} \nabla_{b} J_{\bar{j} b}+\sum_{b} \nabla_{j} \nabla_{b} J_{\bar{i} b}+\sum_{b} \nabla_{\bar{i}} \nabla_{b} J_{j b}\right. \\
& +\sum_{b} \nabla_{\bar{j}} \nabla_{b} J_{i b}-\sum_{a, b}\left(\nabla_{a} J_{\bar{i} a}\right) \nabla_{b} J_{\bar{j} b}-\sum_{a, b}\left(\nabla_{a} J_{\bar{j} b}\right) \nabla_{b} J_{\bar{i} a}+\sum_{a, b}\left(\nabla_{a} J_{i a}\right) \nabla_{b} J_{j b} \\
& \left.+\sum_{a, b}\left(\nabla_{a} J_{j b}\right) \nabla_{b} J_{i a}\right)=0 .
\end{aligned}
$$

Let $(J, g)$ be a critical point of $\mathcal{F}_{\lambda, \mu}$ on $\mathcal{A H}(M)$. Thus, from (3.23), we have further

$$
(\lambda+\mu) \tau-n\left(\lambda \tau+\mu \tau^{*}\right)+\mu \sum\left(2 J_{i a} \nabla_{a} \nabla_{b} J_{i b}+\left(\nabla_{a} J_{i a}\right) \nabla_{b} J_{i b}+\left(\nabla_{a} J_{i b}\right) \nabla_{b} J_{i a}\right)=0 .
$$

Here, we have

$$
\begin{aligned}
& \sum J_{i a} \nabla_{a} \nabla_{b} J_{i b} \\
= & \sum \nabla_{a}\left(J_{i a} \nabla_{b} J_{i b}\right)-\sum\left(\nabla_{a} J_{i a}\right) \nabla_{b} J_{i b} \\
= & -\sum \nabla_{a}\left(J_{i b} \nabla_{b} J_{i a}\right)-\sum\left(\nabla_{a} J_{i a}\right) \nabla_{b} J_{i b} \\
= & -\sum\left(\nabla_{a} J_{i b}\right) \nabla_{b} J_{i a}-\sum J_{i b} \nabla_{a} \nabla_{b} J_{i a}-\sum\left(\nabla_{a} J_{i a}\right) \nabla_{b} J_{i b} \\
= & -\sum J_{i b} \nabla_{b} \nabla_{a} J_{i a}+\sum J_{i b}\left(R_{a b i j} J_{j a}+R_{a b a j} J_{i j}\right) \\
& -\sum\left(\nabla_{a} J_{i a}\right) \nabla_{b} J_{i b}-\sum\left(\nabla_{a} J_{i b}\right) \nabla_{b} J_{i a} \\
= & -\sum J_{i a} \nabla_{a} \nabla_{b} J_{i b}+\tau^{*}-\tau \\
& -\sum\left(\nabla_{a} J_{i a}\right) \nabla_{b} J_{i b}-\sum\left(\nabla_{a} J_{i b}\right) \nabla_{b} J_{i a}
\end{aligned}
$$

and hence,

$$
2 \sum J_{i a} \nabla_{a} \nabla_{b} J_{i b}=\tau^{*}-\tau-\sum\left(\nabla_{a} J_{i a}\right) \nabla_{b} J_{i b}-\sum\left(\nabla_{a} J_{i b}\right) \nabla_{b} J_{i a} .
$$

Thus, from Theorem 3, (3.26) and (3.28), we have the following: 
Theorem 4. Let $(J, g)$ be a critical point of the functional $\mathcal{F}_{\lambda, \mu}$ on $\mathcal{A H}(M)$. Then, we have

$$
\lambda \tau+\mu \tau^{*}=0
$$

From Theorems 3 and 4, taking account of (2.8) and (2.12), we have immediately the following Corollaries 5 and 6 .

Corollary 5. $(J, g) \in \mathcal{A K}(M)$ is a critical point of functional $\mathcal{F}_{-1,1}$ on $\mathcal{A H}(M)$ if and only if $(J, g)$ is a Kähler structure on $M$.

Corollary 6. $(J, g) \in \mathcal{N} \mathcal{K}(M)$ is a critical point of functional $\mathcal{F}_{-1,1}$ on $\mathcal{A H}(M)$ if and only if $(J, g)$ is a Kähler structure on $M$.

Remark 2. The result of Corollary 6 itself is weaker than the one in ([8], Corollary 4).

Remark 3 . Let $M=(M, \Omega)$ be a compact symplectic manifold. We denote by $\mathcal{A K}(M,[\Omega])$ the set of all almost Kähler structures on $M$ with the same Kähler class $[\Omega]$ in the de Rham cohomology group of degree 2. Then in [9], Oguro, Sekigawa, and Yamada showed that $\mathcal{F}_{\frac{1}{2}, \frac{1}{2}}=\frac{4 \pi}{(n-1) !}\left(c_{1} \cdot[\Omega]^{n-1}\right)[M]$ for any $(J, g) \in \mathcal{A K}(M,[\Omega])$, where $c_{1}$ is the first Chern class of $(M, J, g)$. Thus, if $c_{1} \cdot[\Omega]^{n-1} \neq 0$, then there does not exist a critical point in $\mathcal{A} \mathcal{K}(M,[\Omega])$ of the functional $\mathcal{F}_{\frac{1}{2}, \frac{1}{2}}$ on $\mathcal{A H}(M)$.

From Theorem 3 and (3.24), we have also the following.

Theorem 7. Let $(J, g) \in \mathcal{S K}(M)$ be a critical point of the functional $\mathcal{F}_{\lambda, \mu}$ on $\mathcal{A H}(M)$. Then, we have

$$
(\lambda-\mu)\left(\rho_{i j}-\rho_{\overline{i j}}\right)+\mu \sum_{a, b}\left(\left(\nabla_{a} J_{i b}\right) \nabla_{b} J_{j a}-\left(\nabla_{a} J_{\bar{i} b}\right) \nabla_{b} J_{\bar{j} a}\right)=0 .
$$

Corollary 8. Let $(J, g) \in \mathcal{Q K}(M)$ be a critical point of the functional $\mathcal{F}_{\lambda, \mu}$ $(\lambda \neq \mu)$ on $\mathcal{A H}(M)$. Then, $\rho$ is $J$-invariant, and moreover, if $\mu \neq 0, \rho^{*}$ is also $J$-invariant.

Now let $M=(M, J, g)$ be a 4-dimensional Hermitian manifold. Then by (2.4) and (VI), we have the following equalities

$$
\sum_{a}\left(\nabla_{a} J_{i b}\right) \nabla_{b} J_{i a}=0 \text { and } \sum_{a}\left(\nabla_{a} J_{i a}\right) \nabla_{b} J_{i b}=|\omega|^{2} .
$$

Thus, from Theorem 4, (VI) in page 3, and (3.23), we easily have the following.

Theorem 9. Let $M=(M, J, g)$ be a 4-dimensional Hermitian manifold. $(J, g) \in \mathcal{H}(M)$ is a critical point of $\mathcal{F}_{1,-1}$ on $\mathcal{A H}(M)$ if and only if $(J, g)$ is a Kähler structure on $M$. 


\section{Critical points of the functional $\mathcal{F}_{\lambda, \mu}$ on $\mathcal{A H}_{c}(M)$}

In this section, we shall give a condition for an almost Hermitian structure to be a critical points of the functional $\mathcal{F}_{\lambda, \mu}$ on the space $\mathcal{A H}_{c}(M)$ for certain positive constant $c$. Let $(J, g)$ be a critical point of $\mathcal{F}_{\lambda, \mu}$ on $\mathcal{A H}_{c}(M)$. First, consider a deformation $(J(t), g(t))$ of $(J, g)$ of type (i) in Section 3, namely a Blair-Ianus deformation. Then, since $\Omega(t)=\Omega$, we see that $(J(t), g(t)) \in$ $\mathcal{A H}_{c}(M)$ for sufficiently small $|t|$. Thus, applying the similar argument in Section 3 to the present case, we see that the tensor field $T$ defined by (3.18) is $J$-invariant. Next, we consider the deformation $(J(t), g(t))$ in $\mathcal{A H}_{c}(M)$ of $(J, g)$ of type (ii). Since $(J(t), g(t)) \in \mathcal{A H}_{c}(M)$, the 2 -form $A=\left(A_{i j}\right)$ have to satisfy the following equality

$$
\int_{M} \sum_{i j} J_{i j} A_{i j} d v_{g}=0
$$

Further, by taking account of the arguments in Section 3, we see that the 2-form $A$ satisfies the equality (3.20).

Therefore, from (4.1), (3.20) and Lemma 2, by applying the Lagrange's multiplier method, we have finally the following:

Theorem 10. $(J, g)$ is a critical point of the functional $\mathcal{F}_{\lambda, \mu}$ on $\mathcal{A H}_{c}(M)$ if and only if $\lambda \tau+\mu \tau^{*}$ is constant, and $T_{i j}=T_{\bar{i} \bar{j}}, T_{i j}-2 \mu \rho_{i j}^{*}=C g_{i j}$ hold on $M$ with respect to $(J, g)$, where $C=\frac{n-1}{2 n}\left(\lambda \tau+\mu \tau^{*}\right)$.

From Theorem 10, we can easily deduce the following.

Corollary 11. $(J, g)$ is a critical point of the functional $\mathcal{F}_{1,0}$ on $\mathcal{A H}_{c}(M)$ if and only if $(M, J, g)$ is Einstein.

Corollary 11 is the almost Hermitian analogy of the result by Hilbert [5]. From Theorem 10, taking account of (2.10), (2.11), (2.12) and (3.11) in [2], for the critical points $(J, g) \in \mathcal{N} \mathcal{K}(M)$ of the functional $\mathcal{F}_{\lambda, \mu}$ on $\mathcal{A H}_{c}(M)$, we easily have the following.

Corollary 12. Let $(J, g) \in \mathcal{N} \mathcal{K}(M)$ be a critical point of the functional $\mathcal{F}_{\lambda, \mu}(\lambda$ $+\mu=0)$ on $\mathcal{A H}_{c}(M)$ for some positive constant $c$ if and only if

(1) $(J, g)$ is a Kähler structure on $M$, or

(2) $(J, g)$ is an Einstein and $*$-Einstein non-Kähler, nearly Kähler structure on $M$ with $\tau=5 \tau^{*}$.

\section{References}

[1] D. E. Blair and S. Ianus, Critical associated metrics on symplectic manifolds, Nonlinear problems in geometry (Mobile, Ala., 1985), 23-29, Contemp. Math., 51, Amer. Math. Soc., Providence, RI, 1986.

[2] A. Gray, The structure of nearly Kähler manifolds, Math. Ann. 223 (1976), no. 3, $233-248$. 
[3] _ Curvature identities for Hermitian and almost Hermitian manifolds, Tohoku Math. J. (2) 28 (1976), no. 4, 601-612.

[4] A. Gray and L. M. Hervella, The sixteen classes of almost Hermitian manifolds and their linear invariants, Ann. Mat. Pura Appl. (4) 123 (1980), 35-58.

[5] D. Hilbert, Die Grundlagen der Physik, Nachr. Ges. Wiss. Gött. (1915), 395-407.

[6] T. Koda, Critical almost Hermitian structures, Indian J. Pure Appl. Math. 26 (1995), no. 7, 679-690.

[7] S. Koto, Some theorems on almost Kählerian spaces, J. Math. Soc. Japan 12 (1960), 422-433.

[8] T. Oguro and K. Sekigawa, Some critical almost Kähler structures, Colloq. Math. 111 (2008), no. 2, 205-212.

[9] T. Oguro, K. Sekigawa, and A. Yamada, Some critical almost Kähler structures with a fixed Kähler class, Topics in contemporary differential geometry, complex analysis and mathematical physics, 269-277, World Sci. Publ., Hackensack, NJ, 2007.

[10] K. Sekigawa, On some 4-dimensional compact almost Hermitian manifolds, J. Ramanujan Math. Soc. 2 (1987), no. 2, 101-116.

[11] Almost Hermitian manifolds satisfying some curvature conditions, Kodai Math. J. 2 (1979), no. 3, 384-405.

Jung Chan Lee

Department of Mathematics

SUNGKYUNKWAN UNIVERSITY

Suwon 440-746, KoreA

E-mail address: parkj@skku.edu

JEONG HyEONG PARK

Department of Mathematics

SUNGKYUNKWAN UNIVERSITY

SuwON 440-746, Korea

E-mail address: parkj@skku.edu

Kouei Sekigawa

Department of Mathematics

FACUlTy OF SCIENCE

NiIgATA University

Nitgata, 950-2181, JAPAN

E-mail address: sekigawa@math.sc.niigata-u.ac.jp 